\title{
Ruptured ovarian cyst hemorrhage: the swing between conservative and surgical management
}

Sir,

Ruptured ovarian cyst hemorrhage is one of the causes of acute abdomen in women of reproductive age group that can be life threatening. ${ }^{1}$ There have been persistent interest in the discussion of managing ruptured ovarian cyst with or without ovulation. ${ }^{1-4}$ The dilemma of surgical versus conservative/non-surgical management always exist in such cases. It is more of a conundrum when the patient has abnormal coagulation due to either hematological disorders or as a result of anticoagulants. ${ }^{1,5}$ Commonly, indications for surgical interventions are variable and includes: if the source of the bleeding is not confirmed, if patient is hemodynamically unstable, or if there are evidence of ongoing bleeding not settling despite conservative measures in a reasonable time. ${ }^{1}$

There are attempts in literature to retrospectively find predictors for surgical intervention. In one of those studies, a low blood pressure and significant hemoperitoneum on imagining are associated with $66 \%$ chance of the patient requiring a surgical intervention. ${ }^{4}$ In another study, $80 \%$ of their patients were managed surgically. ${ }^{6,7}$

The conservative management in a patient on anticoagulants or with a bleeding disorder should be taking with serious consideration because those women tend to suffer more severe hemorrhage. ${ }^{5}$ The conservative management in those cases entails close observation, periodic repeat hematological investigations, analgesia, and correction of coagulation, replacement of blood and blood products and cardiopulmonary support might be required. ${ }^{1}$ Each of these components of care carries issues with it. The interval of periodic repeat of hemoglobin and coagulation markers may be arbitrary but some references suggest interval of 4-6 hours. ${ }^{4}$

The aim is to maintain safety so the patient does not get to the point of hemodynamic instability or drop her hemoglobin significantly before adequate replacement. That is why it was surprising to see that in a case published in your journal by Agrawal et al; that on conservative management, the hemoglobin was allowed to drop from 8 to $4 \mathrm{~g} / \mathrm{dL}$ over 12-hour-period requiring transfusion of blood and blood products. ${ }^{8}$ Although there might be an element of hemodilution, that means she lost about $50 \%$ of her oxygen carrying capacity compared to when she walked in to the hospital. When conservative approach is the management strategy, vigilance is a paramount feature of the treating team. Risks of blood transfusion should be taken seriously in women of reproductive age due to the possibility of developing abnormal antibodies affecting their pregnancy outcome. So; we believe that the shorter duration from onset of bleeding to the intervention, the less the need for blood transfusion.

A third risk with conservative management in patient on anticoagulants is the possible risk of thrombosis when the anticoagulant effect gets reversed. Avoiding thrombosis is the reason whey the patient is on anticoagulant in the first place. Although that needs to happen with the surgical intervention also; but in our experience the duration of holding the anticoagulant with the surgical intervention is much shorter especially with laparoscopic surgical intervention compared to the conservative nonsurgical malmanagement. ${ }^{1}$ Unfortunately there is no studies in the literature to support that. What the literature shows is that there is no difference in the need for blood transfusion between surgical and non-surgical intervention but that is on retrospective studies. ${ }^{4}$

It is understandable that in many instances, the decision for surgical intervention in patients on anticoagulants is taken reluctantly. That is due to the risks associated with surgical intervention. Those risks are of bleeding and the anesthesia risk related to the medical condition for which the patient is on anticoagulant especially if it is a cardiac disease like mitral valve replacement. ${ }^{1,8}$

Beside those risks related to surgery; there is a risk of compromising future fertility by tending to remove the ovary. ${ }^{4}$ Performing oophorectomy is technically easier than ovarian cystectomy. Another tempting factor about oophorectomy is that the risk of surgical bleeding with it is less than with cystectomy when and if required.

So weighing the risks and benefits of surgical versus conservative management does not depend on the patient factors only but what the health institution is able to offer the patient in-terms of anesthesia, laparoscopic expertise and blood products support. ${ }^{7}$ Surgical intervention is not without risks but it is a fact that when done in a stable patient and it is a laparoscopic intervention by an experienced surgeon the outcome is excellent.

It was not clear in the mentioned case if the hospital where the patient was cared for, had the laparoscopic abilities because the authors concluded that conservative 
management is better compared to laparotomy. ${ }^{8}$ Authors wanted to share and discuss different aspects of management of these cases because it is a common scenario in our practice and our patients outcome so far is good. ${ }^{1,9,10}$

\section{Maryam Al-Shukri*}

Department of Obstetrics and Gynecology, College of Medicine, Sultan Qaboos University, Muscat, Oman

*Correspondence: Dr. Maryam Al-Shukri,

E-mail: mnalshukri@gmail.com

\section{REFERENCES}

1. Al Ghafri W, Gowri V, Al-Khaduri M, Al-Shukri M. Life threatening corpus luteal hemorrhage. Gynecol. 2013;1(1):2.

2. Gupta N, Dadhwal V, Deka D, Jain SK MS. Corpus luteum hemorrhage: rare complication of congenital and acquired coagulation abnormalities. $\mathbf{J}$ Obs Gynecol Res. 2007;33(3):376-80.

3. Payne J, Maclean R, Hampton K, Baxter A, Makris M. Haemoperitoneum associated with ovulation in women with bleeding disorders: the case for conservative management and the role of the contraceptive pill. Hemophilia. 2007;13(1):93-7.

4. Kim JH, Lee SM, Lee J, Jo YR, Moon MH, Shin J, et al. Successful conservative management of ruptured ovarian cysts with hemoperitoneum in healthy women. PLoS One. 2014;9(3):1-5.
5. Jamal A, Mesdaghinia S. Ruptured corpus luteum cysts and anticoagulant therapy. Int J Gynaecol Obs. 2002;(76):319-20.

6. Fiaschetti V, Ricci A, Scarano AL, Liberto V, Citraro D, Arduini S, et al. Hemoperitoneum from corpus luteal cyst rupture: a practical approach in emergency room. Case Rep Emerg Med. 2014;2014:5.

7. Ho W-K, Wang Y-F, Wu H-H, Tsai H-D, Chen T-H, Chen M. Ruptured corpus luteum with hemoperitoneum: case characteristics and demographic changes over time. Taiwan J Obstet Gynecol. 2009;48(2):108-12.

8. Agarwal M, Prybot JE, Dhirasaria A. Ruptured corpus luteum cyst in women on anticoagulant: conservative or surgical management a clinical dilemma. Int J Reprod Contracept Obstet Gynecol. 2017;6(11):5164-5.

9. Mathew M, Al-Ghafri W, Al-Kalbani M, Al-Kharusi L, Gowri V, Al-Shukri M. A clinicopathological study of women with adnexal masses presenting with acute symptoms. Ann Med Health Sci Res. 2014;4(2):286-8.

10. Gowri V, Al Shukri M, Al Khaduri M, Machado L. Clinical and histological profile of surgically managed benign adnexal masses. Oman Med J. 2014;29(3).

Cite this article as: Al-Shukri M. Ruptured ovarian cyst hemorrhage: the swing between conservative and surgical management. Int J Reprod Contracept Obstet Gynecol 2019;8:1706-7. 